TPJU-11/2006

\title{
Large $N$ behavior of two dimensional supersymmetric Yang-Mills quantum mechanics
}

\author{
Maciej Trzetrzelewski * \\ M. Smoluchowski Institute of Physics, Jagiellonian University \\ Reymonta 4, 30-059 Kraków, Poland
}

\begin{abstract}
We analyze the $N \rightarrow \infty$ limit of supersymmetric Yang-Mills quantum mechanics (SYMQM) in two spacetime dimensions. To do so we introduce a particular class of $S U(N)$ invariant polynomials and give the solutions of 2D SYMQM in terms of them. We conclude that in this limit the system is not fully described by the single trace operators $\operatorname{Tr}\left(a^{\dagger^{n}}\right)$ since there are other, bilinear operators $\operatorname{Tr}^{n}\left(a^{\dagger} a^{\dagger}\right)$ that play a crucial role when the hamiltonian is free.
\end{abstract}

PACS: $11.10 . \mathrm{Kk}, 04.60 . \mathrm{Kz}$

Keywords: supersymmetry, quantum mechanics, large N limit

\section{Introduction}

Since the B.F.S.S matrix conjecture [1] relating M-theory with supersymmetric Yang-Mills quantum mechanics in $\mathrm{D}=9+1$ dimensions, there has been a lot of interest in solving the above quantum mechanical systems and their lower dimensional relatives. They are governed by the hamiltonian [2]

$$
H=\frac{1}{2} \pi_{a}^{i} \pi_{a}^{i}+\frac{1}{4} g^{2}\left(f_{a b c} x_{b}^{i} x_{c}^{j}\right)^{2}+H_{F},
$$

*trzetrzelewski@th.if.uj.edu.pl 
where $a, b, c=1, \ldots, N^{2}-1$ ale color indices of $S U(N)$ group, $i, j=1, \ldots, D-1$ are spatial indices and $H_{F}$ is the fermionic term the details of which will not be explored here. The zero energy states of (1) have been widely investigated in number of papers [3] which mainly focus on the existence and asymptotic form of ground states. In $\mathrm{D}=1+1$ case the exact supermultiplet structure for $S U(2)$ group were known for a long time due to Claudson and Halpern [2] . Later on they were extended for arbitrary $S U(N)$ in purely bosonic sector [4]. It is not surprising that $\mathrm{D}=1+1$ systems are solvable since the quartic potential in (1) vanishes in two dimensional systems. The hamiltonian is not free however due to the Gauss law. In other words all physical states obey the constraint $G_{a}|s\rangle=0$ where $G_{a}$ are the $S U(N)$ generators. There are no exact solutions in higher dimensions but a huge effort has been made to study them numerically. The $\mathrm{D}=3+1$ system in bosonic sector has its origin in zero volume pure Yang-Mills theory [5]. Its up to date study for $S U(2)$ gauge group can be found in [6] where nonperturbative values of the spectrum, the Witten index, the supermultiplet structure and the wave functions are discussed. In $\mathrm{D}=9+1$ even for $S U(2)$ case there are no analogous calculations ( i.e. numerical ones ) due to the high complexity of the system.

In this paper we deal with the large $N$ behavior of the $\mathrm{D}=1+1$ systems hoping the the analogous approach will prove useful in higher dimensions. The approach presented here differs from the ones existing in the literature e.g. [7]. We will prove that in $N \rightarrow \infty$ limit the solutions admit very simple form hence it is possible that the same thing takes place in higher dimensions. We also discuss the recent work by Veneziano and Wosiek [8] where planar quantum mechanics is studied as well although in terms of the different model.

The structure of the paper is the following. In section 2 we review the ClaudsonHalpern-Samuel solution. In sections 3 and 4, using the algebraic approach, we obtain a new set of solutions for which the $N$ dependence is evident so that the large $N$ limit is manageable. In section 5 we discuss the vacuum structure of the models. Finally in section 6 we proceed with the $N \rightarrow \infty$ limit for both free and harmonic oscillator hamiltonians. We also point out the relevance of our solutions in that limit.

\section{Claudson-Halpern-Samuel solutions}

The two dimensional system is described by real bosonic coordinates $x_{a}$ and conjugate momenta $\pi_{a},\left[x_{a}, \pi_{b}\right]=i \delta_{a b}$ and fermionic variables $f_{a}, f_{a}^{\dagger},\left\{f_{a}, f_{b}^{\dagger}\right\}=\delta_{a b}$. The hamiltonian and the supercharges are then $[2]$

$$
H=\frac{1}{2} \pi_{a} \pi_{a}+g x_{a} G_{a}, \quad Q=f_{a} \pi_{a},
$$


where $G_{a}=f_{a b c}\left(x_{b} \pi_{c}-i f_{b}^{\dagger} f_{c}\right)$ is the $S U(N)$ generator, $f_{a b c}$ are $S U(N)$ structure tensors. The physical states $|s\rangle$ are those obeying the gauss law $G_{a}|s\rangle=0$ i.e. they are $S U(N)$ singlets. In this $S U(N)$ invariant subspace the hamiltonian is supersymmetric and free i.e $2 H=\left\{Q, Q^{\dagger}\right\}=\pi_{a} \pi_{a} \equiv \pi^{2}$.

The first solutions and the hole supermultiplet structure were obtained by Claudson and Halpern [2] for $S U(2)$ group. In bosonic sector they are

$$
|k\rangle=\frac{\sin (k r)}{k r}|v\rangle, \quad \pi^{2}|k\rangle=k^{2}|k\rangle, \quad r=\sqrt{x_{a} x_{a}}
$$

where $|v\rangle$ is the vacuum state $Q^{\dagger}|v\rangle=Q|v\rangle=0^{1}$. The generalization of these solutions for arbitrary gauge group $U(N)$ is due to Samuel [4]. The idea is to work with $U(N)$ invariants $\lambda_{i}, i=1, \ldots, N$ i.e.

$$
X=U D U^{-1}, \quad D=\operatorname{diag}\left(\lambda_{1}, \ldots, \lambda_{N}\right),
$$

and search for the solutions of the form $f\left(\lambda_{1}, \ldots, \lambda_{N}\right)$. The eigenequation in this coordinates is then

$$
\pi^{2} f=-\frac{1}{M^{2}} \frac{\partial}{\partial \lambda_{i}} M^{2} \frac{\partial}{\partial \lambda_{i}} f=k^{2} f, \quad M=\prod_{i<j}\left(\lambda_{i}-\lambda_{j}\right),
$$

and the solution is

$$
f\left(\lambda_{1}, \ldots, \lambda_{N}\right)=\frac{1}{M} \exp \left(i \sum_{j} k_{j} \lambda_{j}\right) .
$$

These solutions behave badly as $\lambda_{i}$ approaches $\lambda_{j}, i \neq j$ so one has to consider their superposition ( antisymmetrization )

$$
F\left(\lambda_{1}, \ldots, \lambda_{N}\right)=\frac{1}{N !} \sum_{\sigma \in S_{N}}(-1)^{\operatorname{sgn}(\sigma)} f\left(\lambda_{\sigma(1)}, \ldots, \lambda_{\sigma(N)}\right), \quad \pi^{2} F=k^{2} F .
$$

These solutions are now regular. Note that Samuel's solutions can be generalized making the following anzatz

$$
f\left(\lambda_{1}, \ldots, \lambda_{N}\right)=\frac{1}{M} g\left(\lambda_{1}, \ldots, \lambda_{N}\right) .
$$

The differential equation $\pi^{2} f=k^{2} f$ gives $\nabla^{2} g+k^{2} g=0$ which is the Helmholtz differential equation in $N$ dimensions. It is now evident that Samuel's solutions are special ones for which $g=\exp \left(i \sum_{j} k_{j} \lambda_{j}\right), k^{2}=\sum_{j} k_{j}^{2}$, i.e. they correspond to the plane-wave solutions of the Helmholtz equation. It seems that $|0\rangle$.

${ }^{1}$ In this paper we denote the supersymmetric vacuum as $|v\rangle$ while the Fock vacuum as 
Samuel's solutions provide a natural basis of all the solutions since the superposition $\int h\left(k_{1}, \ldots, k_{N}\right) \exp \left(i \sum_{j} k_{j} \lambda_{j}\right)$ obeys Helmholtz equation as well provided $k^{2}=\sum_{j} k_{j}^{2} .{ }^{2}$ However we will indicate in section 4 that there exist solutions when this does not happen.

Note that in this approach the $N$ dependence is not explicit therefore we change the variables from $\lambda_{k}$ to $\left(X^{k}\right) \equiv \operatorname{Tr}\left(X^{k}\right)$ in the following way. The general solution $\frac{1}{M} g$ has to be antisymmetrized due to the $1 / M$ factor. This makes the solution completely symmetric and therefore it can be expanded in terms of symmetric polynomials $\sum_{i} \lambda_{i}=\operatorname{Tr}(X)=0($ for $S U(N)), \sum_{i} \lambda_{i}^{2}=\operatorname{Tr}\left(X^{2}\right) \equiv\left(X^{2}\right), \sum_{i} \lambda_{i}^{3}=$ $\operatorname{Tr}\left(X^{3}\right) \equiv\left(X^{3}\right)$ etc., so that general solutions can be written as

$$
|s\rangle=\sum c_{i_{2} \ldots i_{N}}\left(X^{2}\right)^{i_{2}} \ldots\left(X^{N}\right)^{i_{N}}|v\rangle
$$

We will now attempt to reconstruct these solutions (i.e. determine the $c_{i_{2} \ldots i_{N}}$ 's ) by algebraic methods. It will soon appear clear that the algebraic approach gives the possibility to study the $N \rightarrow \infty$ limit.

\section{$3 \quad$ An algebraic approach}

In this section we use the following conventions

$$
T_{i} T_{j}=\frac{1}{N} \delta_{i j} \mathbf{1}+\frac{1}{\sqrt{2}}\left(d_{i j k}+i f_{i j k}\right) T_{k},
$$

where $T_{i}$ 's are $s u(N)$ generators in the fundamental representation and $f_{i j k} / d_{i j k}$ are complectly antisymmetric/symmetric structure tensors. Moreover, we do not specify here the representation of momentum $\pi_{a}$ and coordinate $x_{a}$ operators. All we need is their commutation relation $\left[x_{a}, \pi_{b}\right]=i \delta_{a b}$.

Note that the conventions that we use differ from the common ones appearing in the literature, namely

$$
T_{i} T_{j}=\frac{2}{N} \delta_{i j} \mathbf{1}+\left(d_{i j k}+i f_{i j k}\right) T_{k}
$$

There is a technical reason for doing so. First, if we define $\left(X^{2}\right)=x_{a} x_{b} \operatorname{Tr}\left(T_{a} T_{b}\right)$ then we have $\left(X^{2}\right)=x_{a} x_{a}$ instead of $\left(X^{2}\right)=2 x_{a} x_{a}$ with an awkward factor of 2 . Moreover the standard identity for $S U(N)$ generators is now

$$
\left[T_{a}\right]_{i j}\left[T_{a}\right]_{k l}=\delta_{i l} \delta_{j k}-\frac{1}{N} \delta_{i j} \delta_{k l}
$$

\footnotetext{
${ }^{2}$ If there are boundary conditions eg. $\operatorname{grad}_{\vec{n}} g=0$ where $\vec{n}$ is a normal vector to the $N-1$ dimensional, closed surface, then the situation is more subtle. It is not clear what, if any, boundary conditions we should take in this case therefore we do not discuss it.
} 
instead of

$$
\left[T_{a}\right]_{i j}\left[T_{a}\right]_{k l}=2 \delta_{i l} \delta_{j k}-\frac{2}{N} \delta_{i j} \delta_{k l} .
$$

We shall also use the notation

$$
\left(T_{a} T_{b} \ldots\right) \equiv \operatorname{Tr}\left(T_{a} T_{b} \ldots\right), \quad(A B \ldots)=A_{a} B_{b} \ldots\left(T_{a} T_{b} \ldots\right)
$$

Let us consider the most general form of the $S U(N)$ invariant eigenstates in purely bosonic sector

$$
|s\rangle=\sum T_{a c b \ldots} x_{a} x_{b} x_{c} \ldots|v\rangle,
$$

where $T_{a b c \ldots}$ is some $S U(N)$ invariant tensor made out of $S U(N)$ tensors $f_{i j k}, d_{i j k}$ and $\delta_{i j}$. The contractions between these structure tensors may be arbitrary so the best way to imagine them is to work in Cvitanovič notation [9] (figure 1)
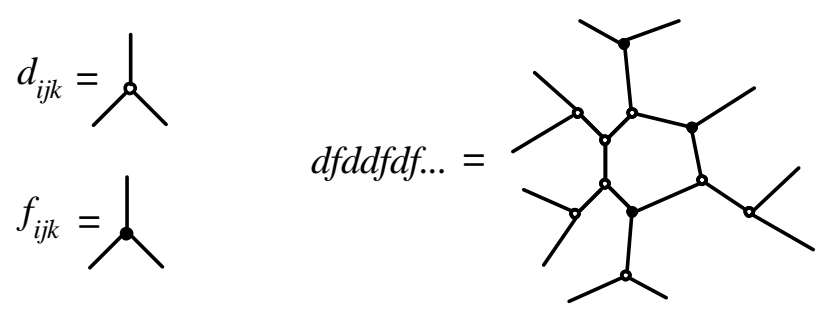

Figure 1: $d_{i j k}, f_{i j k}$ diagrams and a typical tensor diagram.

Each tensor is now represented by a diagram which in general has many loops. One can prove that these loops are not present [10] i.e. they are linear combination of trees. Tree diagram may be expressed in terms of products of trace tensors $\operatorname{Tr}\left(T_{a} T_{b} \ldots\right)$ so if one introduces the following matrix $X=x_{a} T_{a}$ and $\left(X^{k}\right) \equiv$ $\operatorname{Tr}\left(X^{k}\right)$ then using the Cayley-Hamilton theorem for matrices $X$, Eqn. (3) becomes

$$
|s\rangle=\sum c_{i_{2} \ldots i_{N}}\left(X^{2}\right)^{i_{2}} \ldots\left(X^{N}\right)^{i_{N}}|v\rangle
$$

which coincides with (2) as it should.

If $|s\rangle$ is an eigenstate of the hamiltonian $H=\frac{1}{2} \pi^{2}$ then the coefficients $c_{i_{2} \ldots i_{N}}$ can be determined with use of the following commutation relations ( see Appendix A )

$$
\begin{gathered}
{\left[\pi^{2},\left(X^{2}\right)^{n}\right]=-4 i n\left(X^{2}\right)^{n-1}(X \pi)-2 n\left(2 n+1+N^{2}-4\right)\left(X^{2}\right)^{n-1},} \\
{\left[\pi^{2},\left(X^{k}\right)\right]=-2 i k\left(X^{k-1} \pi\right)-k\left(2 N-\frac{k-1}{N}\right)\left(X^{k-2}\right)-\epsilon k \sum_{j=2}^{k-4}\left(X^{k-2-j}\right)\left(X^{j}\right),}
\end{gathered}
$$


where $\epsilon=0$ for $k=3,4,5$ and $\epsilon=1$ for $k>5$. Using (5) and (6) one can prove ( see Appendix B ) that there exist polynomials $P_{k} \equiv P_{k}\left(\left(X^{2}\right),\left(X^{3}\right), \ldots,\left(X^{k}\right)\right)$ of order $\mathrm{k}$ in variables $x_{a}$ such that ${ }^{3}$

$$
\left[\pi^{2}, P_{k}\right]=-2 i \partial_{a} P_{k} \pi_{a}, \quad P_{k} \underset{N \longrightarrow \infty}{\longrightarrow}\left(X^{k}\right), \quad x_{a} \partial_{a} P_{k}=k P_{k}, \quad \Delta P_{k}=0, \quad k=3,4, \ldots
$$

The first few $P_{k}$ 's are

$$
P_{3}=\left(X^{3}\right), \quad P_{4}=\left(X^{4}\right)-\frac{4 N-\frac{9}{2 N}}{N^{2}+1}\left(X^{2}\right)^{2} .
$$

Using (5) and (7) we have

$$
\begin{gathered}
\pi^{2}\left(X^{2}\right)^{n}|v\rangle=-2 n\left(2 n+1+N^{2}-4\right)\left(X^{2}\right)^{n-1}|v\rangle, \\
\pi^{2} P_{k}\left(X^{2}\right)^{n}|v\rangle=-2 n\left(2 n+1+N^{2}-4+2 k\right) P_{k}\left(X^{2}\right)^{n-1}|v\rangle,
\end{gathered}
$$

therefore we obtain a class of solutions ( up to the normalization factor )

$$
\begin{gathered}
|p\rangle=\frac{1}{p r} \sin _{N^{2}-4}(p r)|v\rangle, \quad \pi^{2}|p\rangle=p^{2}|p\rangle, \\
|p\rangle_{k}=\frac{P_{k}}{p r} \sin _{N^{2}-4+2 k}(p r)|v\rangle, \quad \pi^{2}|p\rangle_{k}=p^{2}|p\rangle_{k},
\end{gathered}
$$

where

$$
r \equiv \sqrt{x_{a} x_{a}}=\sqrt{\left(X^{2}\right)}
$$

and

$$
\sin _{t}(x)=\sum_{k=0}^{\infty} \frac{x^{2 k+1}}{1 \cdot 2(3+t) 4(5+t) \ldots 2 k(2 k+1+t)}
$$

\section{Recurrence relations and initial conditions}

Solutions $(8,9)$ are not general ones since we see from equation (4) that the general solution may have arbitrary powers of $\left(X^{k}\right)$ operators which is not the case in $(8,9)$. This problem is not present for $S U(2)$ group since then the differential

\footnotetext{
${ }^{3}$ The order of the polynomial is understood here as the maximal number of times one has to differentiate the polynomial to make it vanish. For example polynomial $x_{1} x_{2} x_{3}$ is of degree 3 although all the variables have degree 1 independently.
} 
eigenequation is with respect to only one variable and the Claudson-Halpern solutions are already the general ones. Indeed we confirm that with algebraic approach since for $S U(2)$ the general form of a solution is

$$
|p\rangle=\sum_{n} a_{n}\left(X^{2}\right)^{n}|v\rangle, \quad \pi^{2}|p\rangle=p^{2}|p\rangle,
$$

Applying (5) to (10) we obtain the following recurrence for $a_{n}$

$$
p^{2} a_{n-1}=-a_{n} 2 n(2 n+1) \Rightarrow a_{n}=(-1)^{n} \frac{a_{0} p^{2 n}}{(2 n+1) !} .
$$

Introducing $r^{2}=\left(X^{2}\right)$ we obtain the Claudson-Halpern solution

$$
|p\rangle=a_{0} \frac{1}{p r} \sin (p r)|v\rangle,
$$

with the normalization factor $a_{0}$.

Let us now apply the same method for the $S U(3)$ group. The general solution is

$$
|p\rangle=\sum_{n} a_{n m}\left(X^{2}\right)^{n}\left(X^{3}\right)^{m}|v\rangle, \quad \pi^{2}|p\rangle=p^{2}|p\rangle .
$$

Applying $(5,6)$ to $(11)$ we get two recurrence relations for $a_{n m}$

4

$$
\begin{gathered}
p^{2} a_{n-1, m}=-2 n(2 n+1+6 m+5) a_{n, m}, \quad m=0,1, \\
p^{2} a_{n-1, m}=-2 n(2 n+1+6 m+5) a_{n, m}-\frac{2}{3}(m+1)(m+2) a_{n-3, m+2} \quad m>1 .
\end{gathered}
$$

Note that (12) already gives us two independent, diagonal in $m$, solutions namely

$$
\left|p_{2}\right\rangle=\frac{a_{00}}{p_{2} r} \sin _{5}\left(p_{2} r\right)|v\rangle, \quad \pi^{2}\left|p_{2}\right\rangle={p_{2}}^{2}\left|p_{2}\right\rangle,
$$

\footnotetext{
${ }^{4}$ To derive (13) we used the identity $\pi^{2}\left(X^{2}\right)^{n}\left(X^{3}\right)^{m}|v\rangle=-2 n(2 n+1+6 m+5)\left(X^{2}\right)^{n-1}\left(X^{3}\right)^{m}|v\rangle-\frac{2}{3} m(m-1)\left(X^{2}\right)^{n+2}\left(X^{3}\right)^{m-2}|v\rangle$, which follows from $(29)$ and $\left(X X T_{a}\right)\left(X X T_{a}\right)=\left(X^{4}\right)-\frac{1}{3}\left(X^{2}\right)^{2}=\frac{1}{6}\left(X^{2}\right)^{2}$.
} 
and

$$
\left|p_{3}\right\rangle=\frac{\left(X^{3}\right) a_{01}}{p_{3} r} \sin _{11}\left(p_{3} r\right)|v\rangle, \quad \pi^{2}\left|p_{3}\right\rangle=p_{3}^{2}\left|p_{3}\right\rangle .
$$

They are exactly the solutions $(8,9)$ for $S U(3)$. In terms of 2 D lattice where each point $(n, m)$ represents a vector $\left(X^{2}\right)^{n}\left(X^{3}\right)^{m}|v\rangle$ it means that the solutions $\left|p_{2}\right\rangle$, $\left|p_{3}\right\rangle$ exist on two rows $(n, 0)$ and $(n, 1)$. The recurrence (13) is represented in figure 2 .

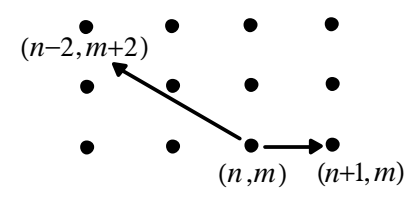

Figure 2: The structure of recurrence (13).

As already indicated the solutions $(8,9)$ are not general ones. It follows that if one fixes e.g. the set $A_{2}=\left\{a_{n 2}, n>0\right\}$ or the set $A_{3}=\left\{a_{n 3}, n>0\right\}$ then (13) determines all $a_{n, 2 m}, m>0$ or $a_{n, 2 m+1}, m>0$ respectively. We will denote the solutions corresponding to these coefficients as $\left|p_{A_{2}}\right\rangle,\left|p_{A_{3}}\right\rangle$ (figure 3 ). Moreover we see that the solutions $(8,9)$ correspond to trivial initial conditions i.e. to $a_{n 2}=$ $a_{n 3}=0$ far all $n$. The general solution is now

$$
\left|p_{1}\right\rangle+\left|p_{2}\right\rangle+\left|p_{A}\right\rangle+\left|p_{B}\right\rangle
$$

with the energy

$$
p_{1}^{2}+p_{2}^{2}+p_{A_{2}}^{2}+p_{A_{3}}^{2}
$$

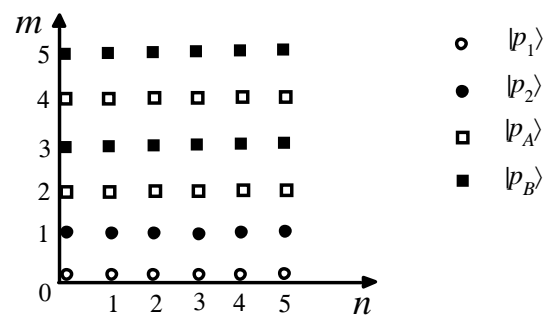

Figure 3: Four independent solutions represented on lattice.

The case of $S U(N)$ is analogous. There are solutions $(8,9)$ corresponding to 
trivial initial condition ${ }^{5}$ and other solutions that require to set the infinite amount of initial coefficients for the recurrence analogous to (13).

Let us note that Samuel's solutions require to set the infinite amount of coefficients. Only then it is possible to obtain the plane wave from the corresponding recurrence. It follows that there are plenty of solutions, e.g. Eqns $(8,9)$, that cannot be obtained from the superposition of Samuel's solutions.

\section{$5 \quad$ Vacuum states}

Here we construct the supersymmetric vacuum state $|v\rangle: Q^{\dagger}|v\rangle=Q|v\rangle=0$ in several fermion sectors. For $n_{F}=0$ sector the general form of the vacuum state is

$$
|v\rangle=\sum c_{i_{2} i_{3} \ldots i_{N}}\left(a^{\dagger^{2}}\right)^{i_{2}}\left(a^{\dagger^{3}}\right)^{i_{3}} \ldots\left(a^{\dagger^{N}}\right)^{i_{N}}|0\rangle, \quad Q^{\dagger}|v\rangle=0,
$$

where $|0\rangle$ is the Fock vacuum and $\left(a^{\dagger^{k}}\right)=\operatorname{Tr}\left(a^{\dagger^{k}}\right), a^{\dagger}=a_{b}^{\dagger} T_{b}$. The supersymmetric generator $Q^{\dagger}$ in terms of creation and annihilation operators is $i \sqrt{2} Q^{\dagger}=$ $\left(a f^{\dagger}\right)-\left(a^{\dagger} f^{\dagger}\right)$ therefore the condition for vacuum becomes

$$
\left(a f^{\dagger}\right)|v\rangle=\left(a^{\dagger} f^{\dagger}\right)|v\rangle .
$$

The last equation can be satisfied only if

$$
|v\rangle=\sum_{k=0}^{\infty} c_{k}\left(a^{\dagger^{2}}\right)^{k}|0\rangle .
$$

This can be proved in the following way. Suppose that the vacuum $|v\rangle$ contains traces $\left(a^{k}\right), k>2$. Since $\left(a f^{\dagger}\right)\left(a^{\dagger^{k}}\right)|0\rangle=k\left(f^{\dagger} a^{\dagger^{k-1}}\right)|0\rangle$, the left hand side of (16) will certainly contain traces $\left(f^{\dagger} a^{\dagger^{k-1}}\right)$ which are not present on the right hand side of (16). Therefore there are no traces $\left(a^{\dagger k}\right), k>2$. For $k=2$ the situation is different because the operator $\left(a^{\dagger} f^{\dagger}\right)$ appears in the right hand side of (16). Now it is straightforward to prove that $c_{k}=\frac{1}{(2 k) ! !}$ therefore in $n_{F}=0$ sector there is only one vacuum given by

$$
|v\rangle=\sum_{k=0}^{\infty} \frac{1}{(2 k) ! !}\left(a^{\dagger^{2}}\right)^{k}|0\rangle .
$$

\footnotetext{
${ }^{5}$ There may exist solutions different from $(8,9)$ that also correspond to trivial initial condition. We do not discuss them here.
} 
The above formula generalizes the $S U(2)$ case [11] to arbitrary $S U(N)$. Note however that this state is badly normalized ${ }^{6}$

$$
\langle v \mid v\rangle=\sum_{k=1}^{\infty}\left(1+\frac{N^{2}-3}{2}\right)\left(1+\frac{N^{2}-3}{4}\right) \ldots\left(1+\frac{N^{2}-3}{2 k}\right) .
$$

This is as it should be since the theory is free. It is however the source of some inconsistencies that we will investigate later.

Now, we can construct the remaining vacuum states in $n_{F}>0$ sectors. They are made out of fermion operators $\left(f^{\dagger^{k}}\right)$ acting on the state $|v\rangle$. Most of operators $\left(f^{\dagger}\right)$ vanish. The list of nontrivial and independent ones is ( see Appendix C )

$$
\left(f^{\dagger^{3}}\right), \quad\left(f^{\dagger^{5}}\right), \ldots,\left(f^{\dagger^{2 N-1}}\right) \quad \text { for } S U(N) .
$$

Such operators are nonvanishing, nilpotent and they commute with $p_{a}$ therefore defining

$$
\left|\epsilon_{3}, \ldots, \epsilon_{2 N-1}\right\rangle:=\left(f^{\dagger^{3}}\right)^{\epsilon_{3}}\left(f^{\dagger^{5}}\right)^{\epsilon_{5}} \ldots\left(f^{\dagger^{2 N-1}}\right)^{\epsilon_{2 N-1}}|v\rangle, \quad \epsilon_{i}=0,1
$$

we have

$$
Q^{\dagger}\left|\epsilon_{3}, \ldots, \epsilon_{2 N-1}\right\rangle=Q\left|\epsilon_{3}, \ldots, \epsilon_{2 N-1}\right\rangle=0,
$$

hence $\left|\epsilon_{3}, \ldots, \epsilon_{2 N-1}\right\rangle$ are vacuum states.

We immediately see that the number of such states, in each fermion sector, is given by the generating function

$$
\left(1+t^{3}\right)\left(1+t^{5}\right) \ldots\left(1+t^{2 N-1}\right)=\sum_{i=0}^{N^{2}-1} b_{i} t^{i},
$$

i.e. the number of vacuum states (18) in $n_{F}$ sector is given by $b_{n_{F}}$. We also recognize the polynomial (19) as the generating function for Betti numbers (i.e Pioncaré polynomial ) of $S U(N)$ group manifold [12] while the vacuum states (18) correspond to nontrivial Lie algebra cocycles of $S U(N)$ [14]. A possible relation of this model to Witten's quantum mechanics [13] on $S U(N)$ manifold will be discussed elsewhere. Instead let us return to the non-normalization of the vacuum state. It is a source of some inconsistencies that we will now discuss ${ }^{7}$.

${ }^{6}$ We use the relation analogous to (5) i.e.

$$
\left[\left(a^{2}\right),\left(a^{\dagger^{2}}\right)^{n}\right]=4 n\left(a^{\dagger^{2}}\right)^{n-1}\left(a^{\dagger} a\right)+2 n\left(2 n-2+N^{2}-1\right)\left(a^{\dagger^{2}}\right)^{n-1} .
$$

${ }^{7}$ I thank G. Veneziano for discussions on this subject 
In section 3 we derived the exact formula for the solutions with trivial initial condition Eqns. ( 8,9). Remarkably they do not vanish if we put $p=0$. In fact we obtain

$$
|p=0\rangle_{k}=P_{k}|v\rangle, \quad \pi^{2}|p=0\rangle_{k}=0,
$$

therefore we obtain a countable set of zero energy solutions in sector with no fermions. In other fermion sectors we have the same situations, i.e. the states

$$
|\psi\rangle_{k}=P_{k}\left|\epsilon_{3}, \ldots, \epsilon_{2 N-1}\right\rangle, \quad k>2, \quad \epsilon_{i}=0,1,
$$

are zero energy states as well. Note however that while $\pi^{2}|\psi\rangle_{k}=0$ we also have $Q^{\dagger}|\psi\rangle_{k} \neq 0$ ! It means that the basic theorem in supersymmetry, namely

$$
Q^{\dagger}|v\rangle=0 \Longleftrightarrow H|v\rangle=0,
$$

does not hold for $H=\frac{1}{2} \pi^{2}$ in the $S U(N)$ invariant sector ${ }^{8}$. To be more precise the implication $\Rightarrow$ is formally correct but the converse is not! This inconsistency is due to the bad normalization of the vacuum state. This state to be mathematically correct does not exist in the Hilbert space therefore the proof of the theorem (21), which assumes that $\left\langle v\left|Q Q^{\dagger}\right| v\right\rangle<\infty$, does not hold anymore. The remedy at this point is to compactify $x_{a}$ so that all the states are normalizable. It turns out that then the model discussed admits the topological interpretation. This issues will be discussed elsewhere.

To conclude this section we see that for $S U(N>2)$ there are two classes of zero energy states $|\psi\rangle$ and $|\psi\rangle_{k}, H|\psi\rangle=H|\psi\rangle_{k}=0$ but only the first class corresponds to supersymmetric vacuum i.e $Q^{\dagger}|\psi\rangle=0$ while $Q^{\dagger}|\psi\rangle_{k} \neq 0$.

\section{The large $\mathbf{N}$ limit}

Taking the $N \rightarrow \infty$ limit in Eqns. $(8,9)$ gives

$$
|p\rangle_{k} \underset{N \longrightarrow \infty}{\longrightarrow}\left(X^{k}\right)|v\rangle
$$

${ }^{8}$ For example $k=3$ gives $P_{3}=\left(X^{3}\right)$ then

$$
\pi^{2}\left(X^{3}\right)|v\rangle=-6 i\left(X^{2} \pi\right)|v\rangle=0,
$$

but

$$
Q^{\dagger}\left(X^{3}\right)|v\rangle=-3 i\left(X^{2} f^{\dagger}\right)|v\rangle \neq 0
$$


where the above limit is understood in the sense of norms i.e. $\lim _{N \rightarrow \infty} \||p\rangle_{k} \|=$ $\lim _{N \rightarrow \infty} \|\left(X^{k}\right)|v\rangle \|$. Thus the $N=\infty$ case is obtained by the operators $\left(X^{k}\right)$ acting on supersymmetric vacuum.

Two comments are now in order. Note that these states also have zero energy since in large $N$ limit (20) coincide with (22). Therefore the states from the continuous spectrum collapse into the zero energy states in the $N \longrightarrow \infty$ limit. It is as if the low energy behavior of the finite $N$ system was given by its large $N$ limit. The lack of $p$ dependence on the right hand side of Eqn. (22) is due to the fact that we are considering the $N \rightarrow \infty$ limit of particular solutions. It does not mean that in this limit there are no solutions with $p$ dependence. Such solutions do exist but they correspond to some nontrivial initial conditions ( see section 4). Moreover the $N=\infty$ case describes no more a quantum mechanical system but rather quantum field theory since the number of degrees of freedom is now infinite. It is now tempting to interpret the infinite number of zero energy states (22) as massless states in some emerging quantum field theory, at least we see that in this limit the hamiltonian $H=\frac{1}{2} \pi^{2}$ provides such possibility.

Let us now rewrite conclusion (22) in terms of creation operators acting on the Fock vacuum $|0\rangle$. It requires little work to prove ( see Appendix C) that in the large $N$ limit vectors (22) are given by the linear combinations of states

$$
|n, m\rangle=\left(a^{\dagger^{2}}\right)^{n}\left(a^{\dagger^{m}}\right)|0\rangle \text {. }
$$

This result is important since it means that in the large $N$ limit the passage from coordinate operators to creation operators is not realized simply by the substitution $X \rightarrow a^{\dagger},|v\rangle \rightarrow|0\rangle$. The difference lies in the structure of the vacuum state in Fock space. In recent work on planar quantum mechanics [8] Veneziano and Wosiek argued that the most important states in Fock space are those given by single trace i.e.

$$
|n\rangle=\left(a^{\dagger^{n}}\right)|0\rangle
$$

therefore vectors (24) differ from (23) by the absence of $\left(a^{\dagger^{2}}\right)^{n}$ operators which are exactly due to the vacuum structure in Fock space. If we want to take that into consideration we should rather work with the basis

$$
|n\rangle=\left(a^{\dagger^{n}}\right)|v\rangle
$$

then with (24).

At this stage one can also ask weather the basis (23) is characteristic of the $N \rightarrow$ $\infty$ basis for the free hamiltonian or is it a good basis even if bound states occur. We verify this question explicitly on the example in supersymmetric harmonic 
oscillator. The hamiltonian and the supersymmetric charge are respectively

$$
\begin{gathered}
H=\frac{1}{2}\left\{Q, Q^{\dagger}\right\}=a_{b}^{\dagger} a_{b}+f_{b}^{\dagger} f_{b}=\frac{1}{2}\left(\pi_{b} \pi_{b}+x_{b} x_{b}\right)-\frac{1}{2}\left(N^{2}-1\right)+f_{b}^{\dagger} f_{b}, \\
Q^{\dagger}=f_{b}^{\dagger} \pi_{b}+i f_{b}^{\dagger} x_{b}=\sqrt{2} i f_{b}^{\dagger} a_{b}, \quad Q=-\sqrt{2} i f_{b} a_{b}^{\dagger} .
\end{gathered}
$$

Since the hamiltonian is simply the number of quanta operator the vacuum of the system is the Fock vacuum $|0\rangle$. Let us search for the solutions of the following form

$$
|\psi\rangle=P_{k} \sum_{n=0}^{\infty} c_{i}\left(X^{2}\right)^{n}|0\rangle,
$$

where $P_{k}$ s are polynomials introduced in section 3 and $c_{n}$ s are some coefficients. Using the properties (7) of these polynomials and the fact that $\pi_{a}|0\rangle=i x_{a}|0\rangle$ we find

$H P_{k}\left(X^{2}\right)^{n}|0\rangle=-n\left(2 n+1+N^{2}-4+2 k\right) P_{k}\left(X^{2}\right)^{n-1}|0\rangle+(2 n+k) P_{k}\left(X^{2}\right)^{n}|0\rangle$,

hence we obtain the recurrence

$$
c_{n+1}=\frac{2 n+k-E}{(n+1)\left(2(n+1)+1+N^{2}-4+2 k\right)} c_{n} .
$$

where $E$ is the energy. In the standard fashion we require that $c_{i>n}=0$ in order to make the state $|\psi\rangle$ properly normalized. This brings us to the condition $E=2 n+k$ which is the eigenvalue of $H$ corresponding to the eigenvector

$$
|\psi\rangle=P_{k} H_{n}|0\rangle, \quad H_{n}=\sum_{i=0}^{n} c_{i}\left(X^{2}\right)^{i} .
$$

The leading term in $N$ of $\|\left(X^{2}\right)^{i}|0\rangle \|^{2}$ is proportional to $N^{4 i}$. ${ }^{9}$ On the other hand $c_{i}^{2}$ s are proportional to $1 / N^{4 i}$ therefore all the terms of $H_{n}$ survive the $N \rightarrow \infty$ limit in the sense of their norms hence large $N$ solutions are given by linear combinations of $\left(X^{2}\right)^{n}\left(X^{k}\right)|0\rangle$. Rewriting this conclusion in terms of creation operators acting on Fock vacuum we obtain ( see Appendix D )

$$
|k\rangle=\left(a^{\dagger^{k}}\right)|0\rangle \text {. }
$$

\footnotetext{
${ }^{9}$ The best way to see this is in coordinate representation. We have$$
\|\left(X^{2}\right)^{i}|0\rangle \|^{2}=\int d X\left(X^{2}\right)^{2 i} e^{-\left(X^{2}\right)}=\sqrt{\pi}^{2 i}\left(a^{\frac{1-N^{2}}{2}}\right)^{(2 i)} \underset{N \longrightarrow \infty}{\longrightarrow} \sqrt{\pi}^{2 i} N^{4 i}
$$ 
This is exactly the basis introduced by Veneziano and Wosiek. However it would be to hasty to conclude that such basis should be used in the large $N$ limit whenever bound states occur. This is because the state (26) is only one example of many solutions that appear in supersymmetric harmonic oscillator. Since the hamiltonian is the number of quanta operator, the following states

$$
\left|i_{2}, i_{3}, \ldots, i_{N}\right\rangle=\frac{1}{\mathcal{N}_{i_{2}, i_{3}, \ldots, i_{N}}}\left(a^{\dagger^{2}}\right)^{i_{2}}\left(a^{\dagger^{3}}\right)^{i_{3}} \ldots\left(a^{\dagger^{N}}\right)^{i_{N}}|0\rangle
$$

with the normalization factor $\mathcal{N}_{i_{2}, i_{3}, \ldots, i_{N}}$ are the eigenstates of $H$ with the eigenvalue $2 i_{2}+3 i_{3}+\ldots+N i_{N}$ and (26) corresponds to just one of them. Moreover one can prove (see Appendix D) that in the large $N$ limit these states become orthogonal and that

$$
\mathcal{N}_{i_{2}, i_{3}, \ldots, i_{N}}^{2}=2^{i_{2}} i_{2} ! 3^{i_{3}} i_{3} ! \ldots N^{i_{N}} i_{N} ! N^{2 i_{2}+3 i_{3}+\ldots+N i_{N}}, \quad N \rightarrow \infty .
$$

Therefore in the sector with fixed number of quanta $n_{B}$ the norms of all the states have the same $N$ dependence namely $N^{n_{B}}$. ${ }^{10}$ This result is a little bit surprising since at first sight it seemed that the norms of the single trace states grow faster with $N$ then the norms of any other states. Since this is not true, there has to be some other criterium that distinguishes their role. However this discussion is beyond the scope of this paper.

\section{Summary}

In this paper we attempted to understand the behavior of quantum mechanics based on $S U(N)$ group when $N$ is large but finite. The discussion is far from complete since the models analyzed here are the easiest ones. We have shown that when the hamiltonian is free $H=\frac{1}{2} \pi_{a} \pi_{a}$ then the single trace states occur as the large $N$ limit of certain solutions. However there are also bilinear operators $\left(a^{\dagger} a^{\dagger}\right)^{k}$ which should be taken into consideration as well. Their emergence is due to the structure of the vacuum state in Fock space so it is not clear weather bilinear operators should be included when we are discussing other hamiltonians with more complicated potentials. Let us also note that in the case of supersymmetric harmonic oscillator non of the states from the Fock space are favored. However there is no t'Hooft coupling in this case so it is not a good example to study the $N \rightarrow \infty$ limit.

\footnotetext{
${ }^{10}$ From Eqn. (28) it may seem at first sight that the longest traces admit the additional factor of $N^{i_{N}}$. This is however an illusion. If the state has $n_{B}$ number of quanta then $i_{k} \mathrm{~s}$ are fixed and do not depend on $N$, moreover starting from $k>n_{B}$ we have $i_{k}=0$
} 
Another interesting issue is the supermultiplet structure which we did not discuss here at all and which certainly simplifies in large $N$ limit.

We also hope that the algebraic approach presented here will be useful while analyzing SYMQM in $\mathrm{D}=4$ and $\mathrm{D}=10$ dimensions. In fact this was our main motivation.

\section{Acknowledgments}

I thank G. Veneziano and J. Wosiek for the discussions. This work was supported by the the grant of Polish Ministry of Science and Education P03B 02427 ( 2004 -2007 ).

\section{Appendix A}

In this appendix we prove (5) and (6). First let us note that if $W$ is an arbitrary function of traces $\left(X^{k}\right)$ then $\left[\pi_{a}, W\right]=-i \partial_{a} W \pi_{a}$ hence

$$
\left[\pi^{2}, W\right]=-2 i \partial_{a}(W) \pi_{a}-\partial_{a} \partial_{a} W .
$$

The proof of (5) is now straightforward. The proof of (6) requires the identity $\left[T_{a}\right]_{i j}\left[T_{a}\right]_{k l}=\delta_{i l} \delta_{j k}-\frac{1}{N} \delta_{i k} \delta_{j l}$ for $S U(N)$ generators. From this identity it follows that

$$
\left(A T_{a} B T_{a}\right)=(A)(B)-\frac{1}{N}(A B),
$$

where $A, B$ are arbitrary matrices. According to (29) we have

$$
\left[\pi^{2},\left(X^{k}\right)\right]=-2 i k\left(X^{k-1} \pi\right)-k \sum_{j=0}^{k-2}\left(T_{a} X^{j} T_{a} X^{k-2-j}\right) .
$$

The last term in (31) is evaluated with use of (30). It is particularly convenient to extract all the $N$ dependence i.e.

$$
\sum_{j=0}^{k-2}\left(T_{a} X^{j} T_{a} X^{k-2-j}\right)=\left(2 N-\frac{k-1}{N}\right)\left(X^{k-2}\right)+\sum_{j=2}^{k-4}\left(X^{k-2-j}\right)\left(X^{j}\right), \quad k>2,
$$

where the sum on the right hand side should not be included when $k<6$. 


\section{Appendix B}

Here we prove the existence of polynomials $P_{k}(7)$ and their properties. First we rewrite (6) in the following form

$$
\begin{gathered}
{\left[\pi^{2}, P_{k}\right]=-2 i \partial_{a} P_{k} \pi_{a}+\tilde{P}_{k-2},} \\
P_{k}=\left(X^{k}\right), \quad \tilde{P}_{k-2}=k\left(2 N-\frac{k-1}{N}\right)\left(X^{k-2}\right)+k \sum_{j=2}^{k-4}\left(X^{k-2-j}\right)\left(X^{j}\right) .
\end{gathered}
$$

$P_{k}$ is of order $k$ in $x_{a}$ variables and $\tilde{P}_{k-2}$ is of order $k-2$ in $x_{a}$.

We now argue that it is possible to add to $P_{k}$ terms in such a way that $\tilde{P}_{k-2}=0$. The proof of this is inductive. First we note that for any polynomial $W_{k-2}$ of order $k-2$, according to (5) we have

$$
\left[\pi^{2},\left(X^{2}\right) W_{k-2}\right]=-2 i \partial_{a}\left(\left(X^{2}\right) W_{k-2}\right) \pi_{a}-2\left(N^{2}-1\right) W_{k-2}-4 x_{a} \partial_{a} W_{k-2}+\left(X^{2}\right) \tilde{W}_{k-4},
$$

where $\tilde{W}_{k-4}=\partial_{a} \partial_{a} W_{k-2}$ is of order $k-4$. Now we note that polynomial $\tilde{P}_{k-2}$ in (31) does not have terms of order lower then $k-2$. This implies that

$$
x_{a} \partial_{a} \tilde{P}_{k-2}=(k-2) \tilde{P}_{k-2},
$$

therefore taking $W_{k-2}=\tilde{P}_{k-2}$ and putting (34) into (33) we obtain

$$
\left[\pi^{2},\left(X^{2}\right) \tilde{P}_{k-2}\right]=-2 i \partial_{a}\left[\left(X^{2}\right) \tilde{P}_{k-2}\right] \pi_{a}-2\left(N^{2}-3+2 k\right) \tilde{P}_{k-2}+\left(X^{2}\right) \tilde{P}_{k-4}^{(1)},
$$

where $\tilde{P}_{k-4}^{(1)}-\partial_{a} \partial_{a} \tilde{P}_{k-2}$ is of order $k-4$. With use of the last equation the $\tilde{P}_{k-2}$ term in (31) can now be subtracted. We have

$$
\left[\pi^{2}, Q^{(1)}\right]=-2 i \partial_{a} Q^{(1)} \pi_{a}+\frac{1}{2\left(N^{2}-3+2 k\right)}\left(X^{2}\right) \tilde{P}_{k-4}^{(1)},
$$

where

$$
Q^{(1)}=P_{k}+\frac{1}{2\left(N^{2}-3+2 k\right)}\left(X^{2}\right) \tilde{P}_{k-2} .
$$

The last term in (36) did not cancel however we see that it also obeys (34) ( i.e if instead of $\tilde{P}_{k}$ in (34) is take $\frac{1}{2\left(N^{2}-3+2 k\right)}\left(X^{2}\right) \tilde{P}_{k-4}^{(1)}$ then (34) will be true ). Therefore we may apply (33) for this term and we obtain

$$
\left[\pi^{2}, Q\right]=-2 i \partial_{a} Q \pi_{a}+\frac{1}{2\left(N^{2}-3+2 k\right)\left(N^{2}-5+2 k\right)}\left(X^{2}\right)^{2} \tilde{P}_{k-6}^{(2)},
$$


where

$$
Q=P_{k}+\frac{1}{2\left(N^{2}-3+2 k\right)}\left(X^{2}\right) \tilde{P}_{k-2}+\frac{1}{4\left(N^{2}-3+4 k\right)\left(N^{2}-5+2 k\right)}\left(X^{2}\right)^{2} \tilde{P}_{k-4}^{(1)},
$$

and $\tilde{P}_{k-6}^{(2)}=\partial_{a} \partial_{a} \tilde{P}_{k-4}$ is of order $k-6$. This inductive procedure gives us the family of polynomials $P_{k-2 i}^{(i-1)}$. Since these polynomials are made out of traces $\left(X^{n}\right)$ and since our group is $S U(N)$ we have $P_{1}^{\left(\frac{k-1}{2}\right)}=0$. Therefore the procedure described here stops only on the polynomial $P_{2}^{\left(\frac{k-2}{2}\right)}=\left(X^{2}\right)$. The remaining term to subtract will be proportional to $\left(X^{2}\right)^{k-1}$ and this can be done using (5).

This ends the proof of the existence of polynomials $P_{k}$ as well as gives the way to construct them. The property $P_{k} \underset{N \longrightarrow \infty}{\longrightarrow}\left(X^{k}\right)$ is evident. The identity $\Delta P_{k}=0$ follows from (29).

\section{Appendix C}

In this appendix we prove (17). We note that since fermion operators $f_{a}^{\dagger}$ anticommute we have

$$
f^{\dagger} f^{\dagger}=\left(f^{\dagger} f^{\dagger} T_{a}\right) T_{a}, \quad f^{\dagger}=f_{a}^{\dagger} T_{a},
$$

therefore

$$
\left(f^{\dagger^{2 n+1}}\right)=\left(f^{\dagger} f^{\dagger} T_{a_{1}}\right) \ldots\left(f^{\dagger} f^{\dagger} T_{a_{n}}\right)\left(T_{a_{1}} \ldots T_{a_{n}} F\right) .
$$

Since operators $\left(f^{\dagger} f^{\dagger} T_{a_{k}}\right)$ commute we may symmetrize over indices

$$
\left(f^{\dagger^{2 n+1}}\right)=\frac{1}{n !}\left(f^{\dagger} f^{\dagger} T_{a_{1}}\right) \ldots\left(f^{\dagger} f^{\dagger} T_{a_{n}}\right)\left(T_{\left(a_{1}\right.} \ldots T_{\left.a_{n}\right)} F\right) .
$$

Generators $T_{a}$ are $N \times N$ matrices therefore according to Cayley-Hamilton theorem if $n \geq N$ then matrix $T_{\left(a_{1}\right.} \ldots T_{\left.a_{n}\right)}$ can be expressed as a linear combination of products of matrices $\left.T_{\left(a_{1}\right.} \ldots T_{a_{k}}\right), k<n^{11}$. This implies that operators $\left(f^{f^{2 n+1}}\right)$, $n \geq N$ can be expressed as a linear combination of products of operators $\left(f^{\dagger^{2 n+1}}\right)$, $n<N$. In fact we have proven something even more general i.e. that for fermionic matrices $f^{\dagger}$ there is a Cayley-Hamilton theorem expressing $f^{\dagger^{2 n+1}}, n \geq N$ in terms of $f^{\dagger^{2 n+1}}, n<N$.

\footnotetext{
${ }^{11}$ In this way Cayley-Hamilton theorem provides a number of identities for structure tensors which are very well known [15]. For a compilation of identities involving invariant tensors see [16]
} 


\section{Appendix D}

Here we prove Eqns. (23), (26) and (28). In order to prove (23) we note that

$$
\left(X^{k}\right)\left(a^{\dagger^{2}}\right)^{n}|0\rangle=\sum_{i_{k}, j_{k}}\left(a^{i_{1}} a^{\dagger^{j_{1}}} \ldots a^{i_{n}} a^{\dagger_{n}}\right)\left(a^{\dagger^{2}}\right)^{n}|0\rangle,
$$

however if $A$ is an arbitrary operator valued matrix and $k, n=0,1,2, \ldots$ then

$$
\begin{gathered}
\left(A a a^{\dagger^{k}}\right)\left(a^{\dagger^{2}}\right)^{n}|0\rangle=\sum_{j=0}^{k-1}\left(A T_{i} a^{\dagger^{j}} T_{i} a^{\dagger^{k-2-j}}\right)\left(a^{\dagger^{2}}\right)^{n}|0\rangle+2 n\left(A a^{\dagger^{k}}\right)\left(a^{\dagger^{2}}\right)^{n-1}|0\rangle \underset{N \longrightarrow \infty}{\longrightarrow} \\
\underset{N \longrightarrow \infty}{\longrightarrow} N\left(A a^{\dagger^{k-1}}\right)\left(a^{\dagger^{2}}\right)^{n}|0\rangle,
\end{gathered}
$$

therefore in the large $N$ limit the leading term of $\left(A a a^{\dagger^{k}}\right)\left(a^{\dagger^{2}}\right)^{n}|0\rangle$ is

$$
\left(A a^{\dagger^{k-1}}\right)\left(a^{\dagger^{2}}\right)^{n}|0\rangle \text {. }
$$

Since A is arbitrary the leading term of $\left(X^{k}\right)\left(a^{\dagger^{2}}\right)^{n}|0\rangle$ is $\left(a^{\dagger^{k}}\right)\left(a^{\dagger^{2}}\right)^{n}|0\rangle$.

Eqn. (26) is surprising since there are no bilinear operators $\left(a^{\dagger} a^{\dagger}\right)$ but indeed it is true. Let us start with the simplest, illustrative case

$$
\left(X^{2}\right)|0\rangle=\frac{1}{2}\left(\left(a^{\dagger^{2}}\right)+N^{2}-1\right)|0\rangle
$$

therefore the norm is

$$
\left\langle 0\left|\left(X^{2}\right)^{2}\right| 0\right\rangle \underset{N \longrightarrow \infty}{\longrightarrow} \frac{1}{4} N^{4} .
$$

Note that the $N^{4}$ dependence does not come from the norm of the state $\left(a^{\dagger^{2}}\right)|0\rangle$ but as a square on $N^{2}$ i.e. from the vacuum state. This picture continues. Let us take

$$
\left(X^{2}\right)^{k}|0\rangle=\frac{1}{2^{k}}\left(\left(a^{\dagger^{2}}\right)+\left(a^{2}\right)+\left(a^{\dagger} a\right)+N^{2}-1\right)|0\rangle
$$

and expand it in terms of powers of $\left(a^{\dagger^{2}}\right),\left(a^{2}\right),\left(a^{\dagger} a\right)$. There is one term which does not have any of those operators namely $\frac{1}{2^{k}}\left(N^{2}-1\right)^{k}$. We now argue that it is leading in $N$.

The operators $\left(a^{\dagger} a\right)$ count only the number of quanta so after commuting them in front of $|0\rangle$ we do not get any additional factors dependent on $N$. Therefore we are left with various powers of operators $\left(a^{\dagger^{2}}\right)$ and $\left(a^{2}\right)$. In order to commute $\left(a^{2}\right)$ in front of $|0\rangle$ we use the relation

$$
\left[\left(a^{2}\right),\left(a^{\dagger^{2}}\right)^{n}\right]=4 n\left(a^{\dagger^{2}}\right)^{n-1}\left(a^{\dagger} a\right)+2 n\left(2 n-2+N^{2}-1\right)\left(a^{\dagger^{2}}\right)^{n-1} .
$$


Therefore to each such commutation we assign the $N^{2}$ factor. There are no more then $k-1$ such commutations so at most we get the $N^{2 k-2}$ factor. Therefore we can write

$$
\left(X^{2}\right)^{k}|0\rangle \underset{N \longrightarrow \infty}{\longrightarrow} \frac{1}{2^{k}}\left(N^{2}-1\right)^{k}|0\rangle .
$$

In other words, among all the vectors from the Fock space the greatest contribution to the state $\left(X^{2}\right)^{k}|0\rangle$ comes from the Fock vacuum. Therefore the greatest contribution to the state $\left(X^{n}\right)\left(X^{2}\right)^{k}|0\rangle$ comes from $\left(X^{n}\right)|0\rangle$ the leading term of which is $\left(a^{\dagger^{n}}\right)|0\rangle$.

To prove (28) we start with the observation that if

$$
|s\rangle=t_{i_{1} \ldots i_{k}} a_{i_{1}}^{\dagger} \ldots a_{i_{k}}^{\dagger}|0\rangle, \quad t_{i_{1} \ldots i_{k}} \in \mathbf{C}
$$

then

$$
\langle s \mid s\rangle=\sum_{\sigma \in S_{k}} t_{\sigma\left(i_{1}\right) \ldots \sigma\left(i_{k}\right)}^{*} t_{i_{1} \ldots i_{k}} \delta_{\sigma\left(i_{1}\right) i_{1}} \ldots \delta_{\sigma\left(i_{k}\right) i_{k}}=t_{\left(i_{1} \ldots i_{k}\right)}^{*} t_{i_{1} \ldots i_{k}}
$$

In our case we have

$$
|s\rangle=\left(a^{\dagger^{2}}\right)^{i_{2}}\left(a^{\dagger^{3}}\right)^{i_{3}} \ldots\left(a^{\dagger^{N}}\right)^{i_{N}}|0\rangle,
$$

therefore $t_{j_{1} \ldots j_{k}}$ is the product of traces $\left(T_{j_{1}} T_{j_{2}} \ldots T_{j_{k}}\right)$

$t_{j_{1} \ldots j_{k}}=\left(T_{j_{1}} T_{j_{2}}\right) \ldots\left(T_{j_{2 i_{2}+1}} T_{j_{2 i_{2}+2}} T_{j_{2 i_{2}+3}}\right) \ldots\left(T_{j_{2 i_{2}+3 i_{3}+1}} T_{j_{2 i_{2}+3 i_{3}+2}} T_{j_{2 i_{2}+3 i_{3}+3}} T_{j_{2 i_{2}+3 i_{3}+4}}\right) \ldots$.

Among all the permutations in the sum (37) there is exactly one ( the identity $\sigma=i d)$ which, due to Kronecker deltas in (37), contracts $i_{1}$ with $\sigma\left(i_{1}\right)$, $i_{2}$ with $\sigma\left(i_{2}\right)$ etc. This term, as we shall see, gives the contribution to the leading term in $N$. There are other terms with the same $N$ dependence but we will include them later on.

Since $T_{i}^{\prime} s$ are hermitian we have $\left(i_{1} \ldots i_{k}\right)^{*}=\left(i_{k} \ldots i_{1}\right)$ therefore we write

$$
\langle s \mid s\rangle=t_{i_{k} \ldots i_{1}} t_{i_{1} \ldots i_{k}}+\text { (the rest of permutations). }
$$

If we introduce the following notation

$$
s_{k}:=\left(j_{k} \ldots j_{1}\right)\left(j_{1} \ldots j_{k}\right),
$$

then it follows that

$$
t_{i_{k} \ldots i_{1}} t_{i_{1} \ldots i_{k}}=s_{2}^{i_{2}} \ldots s_{k}^{i_{k}} \text {. }
$$

The computation of $s_{k}$ is straightforward. With use of the to the identities

$$
\left(A T_{j}\right)\left(B T_{j}\right)=(A B)-\frac{1}{N}(A)(B), \quad T_{j} T_{j}=\left(N-\frac{1}{N}\right) \mathbf{1},
$$


we have

$$
s_{k}=\left(T_{j_{k}} \ldots T_{j_{1}}\right)\left(T_{j_{1}} \ldots T_{j_{k}}\right)=\left(T_{j_{k}} \ldots T_{j_{2}} T_{j_{2}} \ldots T_{j_{k}}\right)-\frac{1}{N}\left(T_{j_{k}} \ldots T_{j_{2}}\right)\left(T_{j_{2}} \ldots T_{j_{k}}\right) .
$$

The first term on the right hand side of the above equation is

$$
\begin{gathered}
\left(T_{j_{k}} \ldots T_{j_{2}} T_{j_{2}} \ldots T_{j_{k}}\right)=\left(N-\frac{1}{N}\right)\left(T_{j_{k}} \ldots T_{j_{3}} T_{j_{3}} \ldots T_{j_{k}}\right)=\ldots \\
\ldots=\left(N-\frac{1}{N}\right)^{k-2}\left(T_{j_{k}} T_{j_{k}}\right)=\left(N-\frac{1}{N}\right)^{k-1} N .
\end{gathered}
$$

The second term is simply $-\frac{1}{N} s_{k-1}$ therefore

$$
s_{k}=\left(N-\frac{1}{N}\right)^{k-1} N-\frac{1}{N} s_{k-1},
$$

hence in the large $N$ limit we have $s_{k}=N^{k}$. From $(38,39)$ it follows now that

$$
\langle s \mid s\rangle=N^{2 i_{2}+3 i_{3}+\ldots+N i_{N}}+\text { (the rest of permutations). }
$$

Now we will extract from the last term in (41) "(the rest of permutations)" all the other leading terms. Due to the cyclicity of traces for each trace $\left(T_{j_{1}} \ldots T_{j_{k}}\right)$ there are $k$ different permutations that give the same term as for the identity permutation. Therefore if there are $i_{2}$ traces of the length $2, i_{3}$ traces of the length 3 , etc. then there are $2^{i_{2}} 3^{i_{3}} \ldots N^{i_{N}}$ additional permutations that give the leading terms. Moreover, among the group of the traces of the length $k$ we can permute those traces in $i_{k}$ ! ways obtaining additional permutations that give the leading terms. Therefore there are in fact $2^{i_{2}} i_{2} ! 3^{i_{3}} i_{3} ! \ldots N^{i_{N}} i_{N}$ ! leading terms. Each of those terms is equal to the term with $\sigma=i d$ so that they are equal to $N^{2 i_{2}+3 i_{3}+\ldots+N i_{N}}$. Therefore the leading term of (41) is now

$$
\langle s \mid s\rangle=2^{i_{2}} i_{2} ! 3^{i_{3}} i_{3} ! \ldots N^{i_{N}} i_{N} ! N^{2 i_{2}+3 i_{3}+\ldots+N i_{N}}+\text { (the rest of permutations'). }
$$

where the ' means all the permutations but the ones we have just extracted.

It is now sufficient to prove that the last term in (42) "(the rest of permutations')" grows slower with $N$ then $N^{2 i_{2}+3 i_{3}+\ldots+N i_{N}}$. To see this we note that each contraction of one generator $T_{a}$ with another one may give at best the factor of N. This happens only if the contracted generators are next to each other i.e.

$$
\left(A T_{a} T_{a} B\right)=\left(N-\frac{1}{N}\right)(A B) \underset{N \longrightarrow \infty}{\longrightarrow} N(A B),
$$

where $\mathrm{A}$ and $\mathrm{B}$ are arbitrary matrices. If the generators are separated by another matrix $C$ then

$$
\left(A T_{a} C T_{a} B\right)=(A B)(C)-\frac{1}{N}(A C B) .
$$


We see that if $C=1$ then we recover the previous result but when $C \neq 1$ then we do not gain the $N$ factor. Therefore among all the possible permutation in (37) the $\sigma=i d$ and the equivalent ones, that we have extracted, are leading in $N$ because in evaluating the term with $\sigma=i d$ we repeatedly used (43) and never used (44). There are no other terms leading in $N$ since there is at least one contraction of the form (44) with $C \neq 1$ in each term of "(the rest of permutations')". Therefore we finally get

$$
\langle s \mid s\rangle=2^{i_{2}} i_{2} ! 3^{i_{3}} i_{3} ! \ldots N^{i_{N}} i_{N} ! N^{2 i_{2}+3 i_{3}+\ldots+N i_{N}} \quad N \rightarrow \infty .
$$

Finally, prove that the states (27) become orthogonal in $N \rightarrow \infty$ limit. Let us consider two arbitrary states

$$
\begin{aligned}
& \left|i_{2}, i_{3}, \ldots, i_{N}\right\rangle=t_{i_{1} \ldots i_{n_{B}}} a_{i_{1}}^{\dagger} \ldots a_{i_{n_{B}}}^{\dagger}|0\rangle, \\
& \left|j_{2}, j_{3}, \ldots, j_{N}\right\rangle=t_{i_{1} \ldots i_{n_{B}}}^{\prime} a_{i_{1}}^{\dagger} \ldots a_{i_{n_{B}}}^{\dagger}|0\rangle,
\end{aligned}
$$

with the number of quanta $n_{B}$. According to Cauchy-Schwarz inequality we have

$$
\left\langle i_{2}, i_{3}, \ldots, i_{N} \mid j_{2}, j_{3}, \ldots, j_{N}\right\rangle \propto N^{l}, \quad l \leq n_{B} .
$$

We have already shown that the case $l=n_{B}$ corresponds to the norms of states with $n_{B}$ number of quanta. The question remains weather there are scalar products, of two distinct states, that have the same $N$ dependence. Fortunately the answer is no. To see this we write the scalar product as

$$
\left\langle i_{2}, i_{3}, \ldots, i_{N} \mid j_{2}, j_{3}, \ldots, j_{N}\right\rangle=t_{\left(i_{1} \ldots i_{n_{B}}\right)}^{*} t_{i_{1} \ldots i_{n_{B}}}^{\prime} .
$$

Since $t_{\left(i_{1} \ldots i_{n_{B}}\right)}$ and $t_{i_{1} \ldots i_{n_{B}}}^{\prime}$ are now different each term in the sum (46) will have at least one contraction of the form ${ }^{12}$

$$
\begin{gathered}
s_{k}^{p q}=\left(T_{i_{k}} \ldots T_{i_{m_{p-1}+1}}\right) \ldots\left(T_{i_{m_{2}}} \ldots T_{i_{m_{1}+1}}\right)\left(T_{i_{m_{1}}} \ldots T_{i_{1}}\right) \times \\
\left(T_{i_{1}} \ldots T_{i_{n_{1}}}\right)\left(T_{i_{n_{1}+1}} \ldots T_{i_{n_{2}}}\right) \ldots\left(T_{i_{n_{q-1}+1}} \ldots T_{i_{k}}\right) .
\end{gathered}
$$

Naturally, such contractions differ from $s_{k}=s_{k}^{1} 1$ 's therefore we cannot express the leading term of (46) in terms of $s_{k}$ 's like in (39). Moreover, using the identities (40) and performing the same manipulations it follows that

$$
s_{k}^{p q}=N^{k-(p-1)-(q-1)}, \quad N \rightarrow \infty,
$$

\footnotetext{
${ }^{12}$ The best way to read (47) is to start with the $\times$ in the middle and read the left and right hand side which are contracted. The integers $\mathrm{p} / \mathrm{q}$ are the numbers of traces on the left/right hand side of $\times$.
} 
therefore for $p=q=1$ we recover the previous result $s_{k}^{1}{ }^{1}=s_{k}=N^{k}$ but when $p>1$ or $q>1$ then the $\mathrm{N}$ dependence will be slower. Since the leading term of (45) always contains at least one $s_{k}^{p}{ }^{q}$ with $p>1$ or $q>1$ it follows that

$$
\left\langle i_{2}, i_{3}, \ldots, i_{N} \mid j_{2}, j_{3}, \ldots, j_{N}\right\rangle \propto N^{l}, \quad l<k .
$$

The states (45) become orthogonal, in the large $N$ limit, in the following sense. Since

$$
\left\langle i_{2}, i_{3}, \ldots, i_{N} \mid j_{2}, j_{3}, \ldots, j_{N}\right\rangle \propto N^{k}, \quad k<n_{B}, \quad N \rightarrow \infty,
$$

therefore if we define

$$
\left|i_{2}, i_{3}, \ldots, i_{N}\right\rangle^{\prime}=\frac{1}{\sqrt{2^{i_{2}} i_{2} ! 3^{i_{3}} i_{3} ! \ldots N^{i_{N}} i_{N} ! N^{2 i_{2}+3 i_{3}+\ldots+N i_{N}}}}\left|i_{2}, i_{3}, \ldots, i_{N}\right\rangle,
$$

then we have

$$
{ }^{\prime}\left\langle i_{2}, i_{3}, \ldots, i_{N} \mid j_{2}, j_{3}, \ldots, j_{N}\right\rangle^{\prime}=\delta_{i_{2} j_{2}} \delta_{i_{3} j_{3}} \ldots \delta_{i_{N} j_{N}} \quad N \rightarrow \infty .
$$

\section{References}

[1] T. Banks, W. Fischler, S. Shenker and L. Susskind, M Theory As A Matrix Model: A Conjecture, Phys. Rev. D55, (1997), 6189; hep-th/9610043.

[2] M. Claudson and M. B. Halpern, Supersymmetric ground state wave functions, Nucl. Phys. 250 (1985) 689. 6189.

[3] J. Froehlich, J. Hoppe, On Zero-Mass Ground States in Super-Membrane Matrix Models, Comm. Math. Phys. 191, 613-626 ; hep-th/9701119.

J. Hoppe, On the Construction of Zero Energy States in Supersymmetric Matrix Models I, II, III; hep-th/9709132, hep-th/9709217, hep-th/9711033.

M. B. Halpern, C. Schwartz, Asymptotic search for ground states of SU(2) matrix theory, Int. J. Mod. Phys. A13 4367-4408 (1998); hep-th/9712133.

J. Hoppe, Shing-Tung Yau, Absence of Zero Energy States in the Simplest $d=3$ (d=5?) Matrix Models; hep-th/9806152

A. Konechny, On asymptotic Hamiltonian for SU(N) Matrix Theory, JHEP 9810 (1998); hep-th/9805046.

J. Froehlich, G.M. Graf, D. Hasler, J. Hoppe, S.-T. Yau, Asymptotic Form of Zero Energy Wave Functions in Supersymmetric Matrix Models, Nucl.Phys. B567 (2000) 231-248; hep-th/9904182 
M. Bordemann, J. Hoppe, R. Suter, Zero Energy States for SU(N): A Simple Exercise in Group Theory?; hep-th/9909191

J. Hoppe, Asymptotic Zero Energy States for $S U(N$ greater or equal 3); hep-th/9912163.

J. Hoppe, J. Plefka, The Asymptotic Groundstate of SU(3) Matrix Theory; hep-th/0002107.

D. Hasler, J. Hoppe, Asymptotic Factorisation of the Ground-State for SU(N)invariant Supersymmetric Matrix-Models; hep-th/0206043.

[4] S. Samuel, Solutions of Extended Supersymmetric Matrix Models for Arbitrary Gauge Groups, Phys. Lett B411, (1997), 268; hep-th/9705167.

[5] M. Lüscher, Some analityc results concerning the mass spectrum of Yang-Mills gauge theories on a torus, Nucl. Phys. B219 (1983) 233.

M. Lüscher, G. Münster, Nucl. Phys. B232 (1984) 445.

P. Weisz, V. Ziemann, Weak coupling expansion of the low-lying energy values in SU(3) gauge theory on torus, Nucl. Phys. B284 (1987) 157.

[6] M. Campostrini and J. Wosiek, High precision study of the structure of $D=4$ supersymmetric Yang-Mills quantum mechanics, Nucl. Phys. B703 hep-th/0407021.

P. van Baal, The Witten Index Beyond the Adiabatic Approximation, in: Michael Marinov Memorial Volume, Multiple Faces in Quantization and Supersymmetry, eds. M. Olshanetsky and A. Vainshtein (World Scientific, Singapore, 2002), hep-th/0112072

J. Kotański, Energy spectrum and wave-functions of four-dimensional Supersymmetric Yang-Mills Quantum Mechanics for very high cut-offs, hep-th/0607012.

[7] M. B. Halpern, Charles B. Thorn, Large $N$ Matrix Mechanics on the LightCone, Int.J.Mod.Phys. A17 (2002) 1517-1542. 6189.

M. B. Halpern, C. Schwartz, The Algebras of Large $N$ Matrix Mechanics, Int. J. Mod. Phys. A14 3059 (1999); hep-th/9809197

[8] G. Veneziano, J. Wosiek, Planar Quantum Mechanics: an Intriguing Supersymmetric Example, JHEP 0601 (2006) 156, hep-th/0512301.

G. Veneziano, J. Wosiek, Large $N$, Supersymmetry ... and QCD, hep-th/0603045.

[9] P. Cvitanovič, e-Book, Group Theory, http://www.nbi.dk/GroupTheory/.

[10] M. Trzetrzelewski, Reduction of su(N) loop tensors to trees, J. Math. Phys. 46,103512 (2005). math-ph/0505084 
[11] J. Wosiek, Supersymmetric Yang-Mills quantum mechanics in various dimensions, Int. J. Mod. Phys. A20 4484-4491 (2005); hep-th/0410066.

[12] L. J. Boya, The geometry of compact Lie groups, Rep. on Math. Phys. 30 , No. 2, 1991.

[13] E. Witten, Constraints on supersymmetry breaking, Nucl. Phys. B202 (1982), 253.

E. Witten, Supersymmetry and Morse theory, J. Diff. Geo. 17, 661 (1982).

[14] J. A. de Azcarraga, A. J. Macfarlane, A. J. Mountain, J. C. Perez Bueno, Cambridge University, (1998); Invariant tensors for simple groups, Nucl. Phys. B510, 657-687.

[15] A. Sudbery, PHD thesis, Cambridge University, (1970); Computer friendly d-tensor identities for su(N), J. Phys. A23, L705-L709 (1990).

[16] J.A. de Azcrraga, A.J. Macfarlane, Compilation of relations for the antisymmetric tensors defined by the Lie algebra cocycles of su(n), Int. J. Mod. Phys. A16 1377-1405 (2001); math-ph/0006026. 